\title{
Management and outcomes of left ventricular assist device-associated endocarditis: a systematic review
}

\author{
Sinal Patel ${ }^{1}$, Syed Saif Abbas Rizvi ${ }^{1}$, Jae Hwan Choi ${ }^{1}$, Dylan P. Horan ${ }^{1}$, Matthew P. Weber ${ }^{1}$, \\ Elizabeth J. Maynes ${ }^{1}$, Jessica G. Y. Luc ${ }^{2}$, Nana Aburjania ${ }^{3}$, John W. Entwistle ${ }^{1}$, Rohinton J. Morris ${ }^{1}$, \\ Howard T. Massey ${ }^{1}$, Vakhtang Tchantchaleishvili ${ }^{1}$
}

${ }^{1}$ Division of Cardiac Surgery, Thomas Jefferson University, Philadelphia, Pennsylvania, USA; ${ }^{2}$ Division of Cardiovascular Surgery, Department of Surgery, University of British Columbia, Vancouver, British Columbia, Canada; ${ }^{3}$ Division of Infectious Diseases, Thomas Jefferson University, Philadelphia, Pennsylvania, USA

Correspondence to: Vakhtang Tchantchaleishvili, MD. Assistant Professor of Surgery, Division of Cardiothoracic Surgery, Thomas Jefferson University, 1025 Walnut St., Suite 607, Philadelphia, PA 19107, USA. Email: Vakhtang.Tchantchaleishvili@jefferson.edu.

\begin{abstract}
Background: Left ventricular assist device (LVAD)-associated endocarditis remains poorly studied, especially in newer continuous-flow LVADs (CF-LVADs). The aim of this review was to assess outcomes of patients with LVAD-associated endocarditis, as stratified by CF-LVAD and pulsatile LVAD (P-LVAD) use as well as by different interventions and pathogen types.

Methods: An electronic search was performed to identify studies in the English literature on LVADassociated endocarditis.

Results: Overall, 16 articles with 26 patients were included; seven had CF-LVADs and 19 had P-LVADs; time to development of endocarditis was 91 days (152 vs. 65 days, respectively, $\mathrm{P}=0.05$ ). Eleven of 25 patients were treated with antibiotics only. Remaining 14 patients received antibiotics, however, they also underwent additional surgical intervention. One patient was treated with embolization alone for mycotic aneurysm and was therefore excluded. At a median follow-up time of 344 days post implant, there was no difference in overall mortality between CF-LVAD and P-LVAD-associated endocarditis patients (57.9\% vs. $42.9 \%$, $\mathrm{P}=0.81$ ). Patients who underwent additional surgical intervention had higher overall survival compared to those treated with antibiotics alone $(71.4 \%$ vs. $27.3 \%, \mathrm{P}=0.07)$; with no difference in outcomes amongst those who underwent surgical device exchange as compared to heart transplantation $(80.0 \%$ vs. $66.7 \%$; $\mathrm{P}=0.23$ )

Conclusions: Compared to patients with P-LVADs, CF-LVAD patients appeared to be resistant to early development of LVAD-associated endocarditis. There was a trend towards high survival observed amongst patients who underwent additional surgical intervention as compared to those treated with antibiotics alone, with no difference amongst surgical device exchange as compared to heart transplantation. Advantages of additional surgical intervention $v s$. medical therapy alone deserves further exploration to determine its applicability in CF-LVADs.
\end{abstract}

Keywords: Left ventricular assist device (LVAD); infection; endocarditis

Submitted Nov 22, 2018. Accepted for publication Apr 08, 2019.

doi: 10.21037/acs.2019.04.04

View this article at: http://dx.doi.org/10.21037/acs.2019.04.04

\section{Introduction}

Heart transplantation remains the best treatment option for patients with end stage heart failure (1). However, a left ventricular assist device (LVAD) is a viable alternative for hemodynamic improvement in these patients due to a shortage of donor hearts. LVAD use in the management of refractory heart failure as a destination therapy or as a 
bridge to transplantation has gained popularity since its first approval in 1994 and has shown to improve survival and quality of life in these patients (2-5).

Compared to the older pulsatile LVADs (P-LVADs), continuous-flow LVADs (CF-LVADs) are associated with better survival outcomes and infection rates due to their smaller size and simplified pump mechanisms $(6,7)$. However, despite improvements and advancement in CF-LVAD technology, device-specific infection and sepsis remains a major complication in these patients (8). The HeartMate II bridge to transplantation trial and the HeartMate II destination therapy trial have both shown high rates of LVAD-specific infections $(28 \%$ and $35 \%$, respectively) and sepsis (20\% and $36 \%$, respectively) in LVAD patients $(9,10)$.

The majority of LVAD-specific infections consist of driveline infections and pump-pocket infections, while LVAD-associated endocarditis accounts for least amount of all the infections among patients with an LVAD but is associated with high mortality $(11,12)$. Both medical and surgical management are considered viable options for LVAD-associated endocarditis treatment $(13,14)$.

In this systematic review, we sought to assess the outcomes of patients with LVAD-associated endocarditis, with comparison of antimicrobial therapy alone versus additional surgical intervention.

\section{Methods}

\section{Literature search strategy}

Electronic searches were performed in January 2018 using Ovid Medline, PubMed, Cochrane Central Register of Controlled Trials (CCTR), Cochrane Database of Systematic Reviews (CDSR), ACP Journal Club, and Database of Abstracts of Review of Effectiveness (DARE). Maximum sensitivity of the search strategy was achieved by using the following search terms: (heart-assist devices OR ventricular assist device OR assisted circulation) AND (endocarditis OR bacterial endocarditis). References in the retrieved articles were also reviewed for identification of potentially relevant studies and assessed using inclusion and exclusion criteria.

\section{Selection criteria}

Eligible articles for the present systematic review include those that addressed the development of endocarditis in patients with an LVAD; case reports, case series and retrospective studies were included. Duplicate articles were screened out and only the most complete reports were included for quantitative assessment with no overlapping individual patient data. We excluded studies on patients $<18$ years of age, studies not published in the English language and those not involving human subjects. Furthermore, abstracts, conference presentations, editorials, reviews and expert opinions were also excluded.

\section{Definition}

LVAD-associated endocarditis is defined as clinical evidence of pump and/or cannula infection as demonstrated by positive LVAD explanted culture along with the presence of vegetations on echocardiography or a vascular phenomenon as defined by the modified Duke's criteria (15). In this systematic review, the criteria used for diagnosing individual LVAD-associated endocarditis cases as reported by the studies are shown in Table 1.

\section{Data extraction and critical appraisal}

Patient-level data was extracted from article texts, tables and figures. Two investigators independently reviewed each retrieved article (S Patel, JH Choi). Discrepancies between the two reviewers were resolved by discussion and consensus. When data was not available, attempts were made to contact corresponding authors to obtain the relevant data for the current review.

\section{Statistical analysis}

Patient-level data were extracted from selected studies and subject to statistical analysis. Baseline characteristics and demographics were reported using descriptive statistics, including median and interquartile ranges (IQR), rounded to the nearest integer. Primary endpoint of overall survival rates was analyzed between the CF-LVAD and P-LVAD subgroups, as well as between different interventions and pathogen types in LVAD-associated endocarditis.

Continuous variables were compared using Wilcoxon ranksum test, while categorical variables were analyzed using chisquare test. Estimated survival over time was further analyzed using the Kaplan-Meier method in those patients where timeto-event data was available. Missing data were considered missing at random, and patients with missing variables were excluded from analysis of these particular variables. All analyses were performed with R software, version 3.5.1 (R Foundation 


\begin{tabular}{|c|c|c|c|}
\hline Clinical signs + blood culture, n (\%) & $2(28.6)$ & $1(5.3)$ & $3(11.5)$ \\
\hline Clinical signs + explanted LVAD culture, $\mathrm{n}(\%)$ & $1(14.3)$ & $11(57.9)$ & $12(46.2)$ \\
\hline Explanted LVAD culture only, $\mathrm{n}(\%)$ & $0(0.0)$ & $3(15.8)$ & $3(11.5)$ \\
\hline Clinical signs + blood culture + TEE, n (\%) & $1(14.3)$ & $0(0.0)$ & $1(3.8)$ \\
\hline Clinical signs + blood culture + explanted LVAD culture, n (\%) & $0(0.0)$ & $2(10.5)$ & $2(7.7)$ \\
\hline Clinical signs + blood culture + TEE + explanted LVAD culture, $n(\%)$ & $0(0.0)$ & $1(5.3)$ & $1(3.8)$ \\
\hline Clinical signs + TEE + explanted LVAD culture, $n(\%)$ & $0(0.0)$ & $1(5.3)$ & $1(3.8)$ \\
\hline
\end{tabular}

for Statistical Computing, Vienna, Austria). P values $<0.05$ were considered statistically significant.

\section{Results}

\section{Study characteristics}

Overall, 376 records were identified in the literature search. Following application of the inclusion and exclusion criteria, 16 articles from 1997 to 2017 were included for analysis, of which 12 were case reports, one was a case series and three were retrospective studies from different institutions. Out of 26 patients, seven patients had a CF-LVAD and 19 had a P-LVAD implanted. A PRISMA flow diagram depicting the overall search strategy is provided in Figure 1. A manual search of references did not yield further studies.

\section{Baseline demographics}

Baseline demographics of patients with CF-LVAD and P-LVAD implantation are shown in Table 2. Most patients who received an LVAD were male $(57.7 \%)$ with a median age of 51.0 (IQR, 41.5-59.5) years at LVAD implantation. No significant differences were seen between the median age of patients undergoing CF-LVAD placement and P-LVAD placement [CF-LVAD 55.0 (IQR, 39.5-60.0) vs. P-LVAD 50.5 (IQR, 41.8-58.2) years, $\mathrm{P}=0.95]$. Etiology of heart failure was comparable between CF-LVAD and P-LVAD patients.

\section{Infectious characteristics}

The clinical presentation of patients with LVAD-associated endocarditis is shown in Table 3. Overall median duration of LVAD support was 207 (IQR, 79-378) days with no significant difference between the CF-LVAD and P-LVAD group [CF-LVAD 304 (IQR, 204-395) days vs. P-LVAD 184 (IQR, 77-291) days; $\mathrm{P}=0.11$ ]. The median time to endocarditis following LVAD implantation was 91 days. There was a trend for a longer time to endocarditis following LVAD implantation for patients with CF-LVADs [CF-LVAD 152 (IQR, 91-1,422) days vs. P-LVAD 65 (IQR, 55-153) days, $\mathrm{P}=0.05]$. The explanted device culture was obtained in 22 patients. Of these, the exact location of the microbial foci on the explanted device was reported in 13 patients only. Among these 13 patients, inflow valves $(n=4,30.8 \%)$ and outflow grafts $(n=4,30.8 \%)$ were the most common sites of LVAD-associated endocarditis, followed by inflow and outflow valves ( $\mathrm{n}=3,23.1 \%), \mathrm{LVAD}$ diaphragms $(\mathrm{n}=1,7.7 \%)$ and inflow grafts $(\mathrm{n}=1,7.7 \%)$.

\section{Microbiological profile}

The microbiology profile of organisms isolated from device sites and blood is shown in Table 4. Of all the positive cultures among the 26 patients, bacteria were isolated from the explanted LVAD device in $34.6 \%(\mathrm{n}=9)$ and from the bloodstream in $69.2 \%(\mathrm{n}=18)$. Within the isolated bacterial cultures from the explanted device, the most common organism was Pseudomonas aeruginosa $(\mathrm{n}=3,11.5 \%)$ followed by Staphylococcus epidermidis $(\mathrm{n}=2,7.7 \%)$ and Enterococcus $(\mathrm{n}=2,7.7 \%)$, whereas coagulase-negative Staphylococcus ( $\mathrm{n}=5,19.2 \%)$ and methicillin-sensitive Staphylococcus aureus (MSSA) $(\mathrm{n}=3,11.5 \%)$ were the most common isolates from 


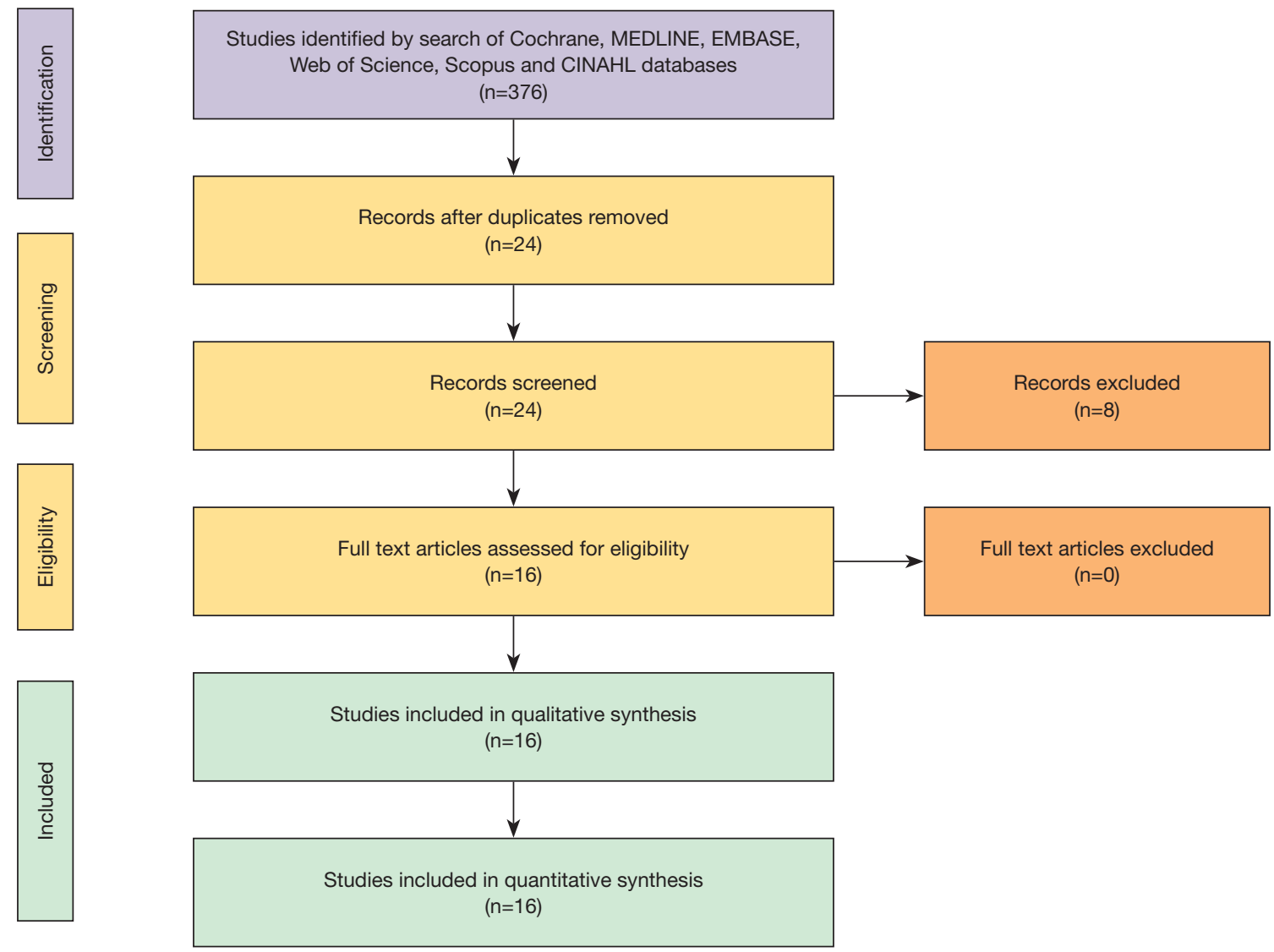

Figure 1 PRISMA schematic of search strategy. PRISMA, preferred reporting items for systematic reviews and meta-analyses.

\begin{tabular}{|c|c|c|c|c|}
\hline Variable & CF-LVAD & P-LVAD & Total & $P$ value \\
\hline Age, median (IQR) (years) & $55.0(39.5-60.0)$ & $50.5(41.8-58.2)$ & $51.0(41.5-59.5)$ & 0.95 \\
\hline Male, $\mathrm{n}(\%)$ & $7(100.0)(n=7)$ & $8(42.1)(n=19)$ & $15(57.7)(n=26)$ & 0.03 \\
\hline Etiology, n (\%) & $(n=5)$ & $(n=7)$ & $(n=12)$ & 0.19 \\
\hline Dilated cardiomyopathy & $1(20.0)$ & $3(42.9)$ & $4(33.3)$ & \\
\hline Ischemic cardiomyopathy & $2(40.0)$ & $2(28.6)$ & $4(33.3)$ & \\
\hline Idiopathic cardiomyopathy & $0(0.0)$ & $2(28.6)$ & $2(16.7)$ & \\
\hline Non-ischemic cardiomyopathy & $2(40.0)$ & $0(0.0)$ & $2(16.7)$ & \\
\hline
\end{tabular}

LVAD, left ventricular assist devices; CF-LVAD, continuous-flow left ventricular assist devices; P-LVAD, pulsatile left ventricular assist devices; IQR, interquartile range.

a patient's bloodstream followed by methicillin-resistant Staphylococcus aureus (MRSA) ( $\mathrm{n}=2,7.7 \%)$, Pseudomonas aeruginosa $(\mathrm{n}=2,7.7 \%)$, and alpha-hemolytic Streptococcus $(\mathrm{n}=2,7.7 \%)$. Similarly, fungal cultures were positive from the explanted device in $19.2 \%(\mathrm{n}=5)$ of patients and from the bloodstream in $15.4 \%(\mathrm{n}=4)$ of all patients. Among fungal isolates, Candida $(\mathrm{n}=2,7.7 \%)$ and Aspergillus $(\mathrm{n}=2$, $7.7 \%$ ) were the two most common organisms isolated from 


\begin{tabular}{|c|c|c|c|c|}
\hline Time to infection, median [IQR] (days) & $152[91-1,422]$ & $65[55-153]$ & $91[57-221]$ & 0.05 \\
\hline Duration of LVAD support, median [IQR] (days) & 304 [204-395] & 184 [77-291] & 207 [79-378] & 0.11 \\
\hline Follow-up time post implant, median [IQR] (days) & $395[350-1,551]$ & 266 [103-859] & $344[170-1,022]$ & 0.10 \\
\hline Inflow valves & $0(0.0)$ & $4(33.3)$ & $4(30.8)$ & \\
\hline Outflow grafts & $1(100.0)$ & $3(25.0)$ & $4(30.8)$ & \\
\hline Inflow and outflow valves & $0(0.0)$ & $3(25.0)$ & $3(23.1)$ & \\
\hline LVAD diaphragms & $0(0.0)$ & $1(8.3)$ & $1(7.7)$ & \\
\hline Clinical manifestations of endocarditis, n (\%) & $(n=7)$ & $(n=16)$ & $(n=23)$ & 0.85 \\
\hline Fever or leukocytosis & $5(71.4)$ & $10(62.5)$ & $15(65.2)$ & \\
\hline Cerebrovascular accident & $1(14.3)$ & $3(18.8)$ & $4(17.4)$ & \\
\hline LVAD outflow obstruction & $1(14.3)$ & $1(6.3)$ & $2(8.7)$ & \\
\hline LVAD inflow obstruction & $0(0.0)$ & $1(6.3)$ & $1(4.3)$ & \\
\hline Cachexia or failure to thrive & $0(0.0)$ & $1(6.3)$ & $1(4.3)$ & \\
\hline Interventions, $\mathrm{n}(\%)^{\star}$ & $(n=6)$ & $(n=19)$ & $(n=25)$ & 0.36 \\
\hline Antibiotics only & 5 (83.3) & $6(31.6)$ & $11(44.0)$ & \\
\hline
\end{tabular}

*, this section is based on 25 patients since one patient was treated with embolization. LVAD, left ventricular assist devices; CF-LVAD, continuous-flow left ventricular assist devices; P-LVAD, pulsatile left ventricular assist devices.

the explanted LVAD devices.

\section{Clinical presentation and management}

No significant differences were noted between CF-LVAD and P-LVAD patients in terms of clinical presentation leading to the diagnosis of LVAD-associated endocarditis $(\mathrm{P}=0.85)$ (Table 3). Major clinical manifestations of LVADassociated endocarditis included fever and leukocytosis in both groups (CF-LVAD: 71.4\% vs. P-LVAD: 62.5\%). Other clinical manifestations included cerebrovascular accidents (CF-LVAD: $14.3 \%$ vs. P-LVAD: 18.8\%), cachexia (CF-
LVAD: $0 \%$ vs. P-LVAD: 6.3\%), LVAD outflow obstruction (CF-LVAD: $14.3 \%$ vs. P-LVAD: 6.3\%), and LVAD inflow obstruction (CF-LVAD: 0\% vs. P-LVAD: 6.3\%). LVADassociated endocarditis was most frequently confirmed by an explanted device culture in P-LVAD patients ( $\mathrm{n}=11,57.9 \%)$ and by the presence of mycotic aneurysm in the CF-LVAD patients ( $\mathrm{n}=3,42.9 \%)$ (Table 1 ).

All patients with LVAD-associated endocarditis were clinically managed with antibiotics alone or with antibiotics and additional surgical intervention. Of all patients in this review, data on the management of LVAD-associated endocarditis was available in 25 patients (CF-LVAD: 6 s. 


\begin{tabular}{|c|c|c|}
\hline Organisms & $\begin{array}{l}\text { Explanted } \\
\text { device culture }\end{array}$ & $\begin{array}{l}\text { Initial blood } \\
\text { culture }\end{array}$ \\
\hline $\begin{array}{l}\text { Bacterial episodes, } \\
\text { n (\%) }\end{array}$ & 9 (34.6) & $18(69.2)$ \\
\hline MRSA & $0(0.0)$ & $2(7.7)$ \\
\hline MSSA & $1(3.8)$ & $3(11.5)$ \\
\hline MRSE & $0(0.0)$ & $1(3.8)$ \\
\hline $\begin{array}{l}\text { Alpha hemolytic } \\
\text { Streptococcus }\end{array}$ & $0(0.0)$ & $2(7.7)$ \\
\hline $\begin{array}{l}\text { Coagulase-negative } \\
\text { Staphylococcus }\end{array}$ & $0(0.0)$ & 5 (19.2) \\
\hline $\begin{array}{l}\text { Staphylococcus } \\
\text { epidermidis }\end{array}$ & $2(7.7)$ & $0(0.0)$ \\
\hline Enterococcus & $2(7.7)$ & $1(3.8)$ \\
\hline Pseudomonas & $3(11.5)$ & $2(7.7)$ \\
\hline Escherichia coli & $1(3.8)$ & $1(3.8)$ \\
\hline Klebsiella & $0(0.0)$ & $1(3.8)$ \\
\hline Fungal episodes, n (\%) & $5(19.2)$ & $4(15.4)$ \\
\hline Candida & $2(7.7)$ & $4(15.4)$ \\
\hline Aspergillus & $2(7.7)$ & $0(0.0)$ \\
\hline Myceliophthora & $1(3.8)$ & $0(0.0)$ \\
\hline Negative, n (\%) & $3(11.5)$ & $4(15.4)$ \\
\hline Unspecified, n (\%) & 9 (34.6) & 5 (19.2) \\
\hline \multicolumn{3}{|c|}{$\begin{array}{l}\text { LVAD, left ventricular assist devices; MSSA, methicillin- } \\
\text { sensitive Staphylococcus aureus; MRSA, methicillin- } \\
\text { resistant Staphylococcus aureus; MRSE, methicillin-resistant } \\
\text { Staphylococcus epidermidis. }\end{array}$} \\
\hline
\end{tabular}

P-LVAD: 19). Of these 25 patients, 11 (44.0\%) were treated with antibiotics alone. The remaining 14 patients underwent further surgical interventions including heart transplantation ( $\mathrm{n}=6,24.0 \%)$, LVAD exchange $(\mathrm{n}=5,20.0 \%)$, LVAD explantation without heart transplantation $(\mathrm{n}=1,4.0 \%)$, inflowoutflow valve exchange $(\mathrm{n}=1,4.0 \%)$ and outflow graft repair $(\mathrm{n}=1,4.0 \%)$ (Table 3). Of note, a higher number of patients in the CF-LVAD group were managed with antibiotics alone (CF-LVAD: $\mathrm{n}=5,83.3 \%$ vs. P-LVAD: $\mathrm{n}=6,31.6 \%$ ).

\section{Outcomes}

Overall survival was $53.8 \%$ with no significant difference

\begin{tabular}{|c|c|c|}
\hline & Survival, n(\%) & r value \\
\hline CF-LVAD vs. P-LVAD & & 0.81 \\
\hline CF-LVAD $(n=7)$ & $3(42.9)$ & \\
\hline P-LVAD $(n=19)$ & $11(57.9)$ & \\
\hline Total $(n=26)$ & $14(53.8)$ & \\
\hline \multicolumn{2}{|l|}{ Bacterial vs. fungal infections } & 0.36 \\
\hline Bacterial $(n=10)$ & $4(40.0)$ & \\
\hline Fungal $(n=5)$ & $4(80.0)$ & \\
\hline Total $(n=15)$ & $8(53.3)$ & \\
\hline \multicolumn{2}{|c|}{ Antibiotics alone vs. surgical intervention } & 0.07 \\
\hline Antibiotics $(n=11)$ & $3(27.3)$ & \\
\hline Surgery $(n=14)$ & $10(71.4)$ & \\
\hline Total $(n=25)$ & $13(52.0)$ & \\
\hline \multicolumn{2}{|c|}{ LVAD exchange vs. heart transplantation } & 0.23 \\
\hline Exchange $(n=5)$ & $4(80.0)$ & \\
\hline Heart transplant $(n=6)$ & $4(66.7)$ & \\
\hline Total $(n=11)$ & $8(72.7)$ & \\
\hline \multicolumn{3}{|c|}{$\begin{array}{l}\text { LVAD, left ventricular assist devices; CF-LVAD, continuous-flow } \\
\text { left ventricular assist devices; P-LVAD, pulsatile left ventricular } \\
\text { assist devices. }\end{array}$} \\
\hline
\end{tabular}

between CF-LVAD and P-LVAD-associated endocarditis (CF-LVAD: $42.9 \%$ vs. P-LVAD: $57.9 \%, \mathrm{P}=0.81$ ) during the median follow-up period of 344 days [CF-LVAD 395 (IQR, 350-1,551) days vs. P-LVAD 266 (IQR, 103-859) days; $\mathrm{P}=0.10$ ] (Tables 3,5). Compared to bacterial infections, no significant difference was noted in survival of patients with fungal infections $(\mathrm{P}=0.36)$ (Table 5). There was a trend towards superior overall survival in patients who underwent surgical intervention in addition to antibiotics for management of LVAD-associated endocarditis compared to those treated with antibiotics alone (surgery and antibiotics: $71.4 \%$ vs. antibiotics alone: $27.3 \%, \mathrm{P}=0.07$ ) (Table 5). However, Kaplan-Meier analysis did not detect a statistical significance among the two groups $(\mathrm{P}=0.17)$ (Figure 2). Within the surgery subgroup, no significant difference in overall survival was observed between the patients who underwent device exchange and patients who 


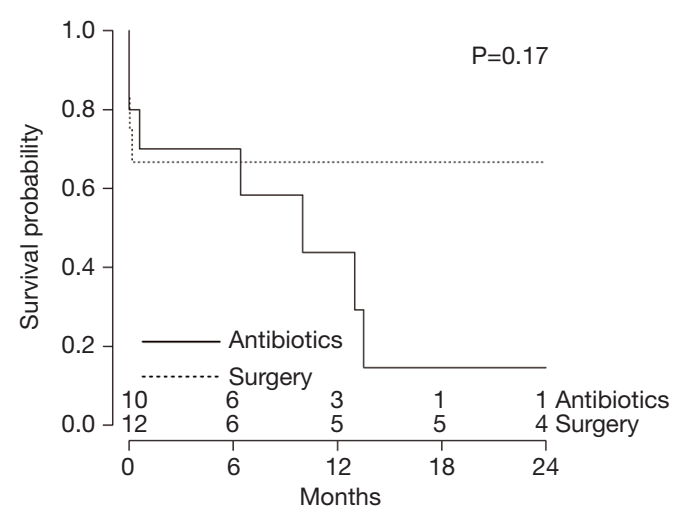

Figure 2 Kaplan-Meier survival analysis of patients with LVADassociated endocarditis who underwent surgical intervention as compared to antibiotics treatment only. LVAD, left ventricular assist devices.

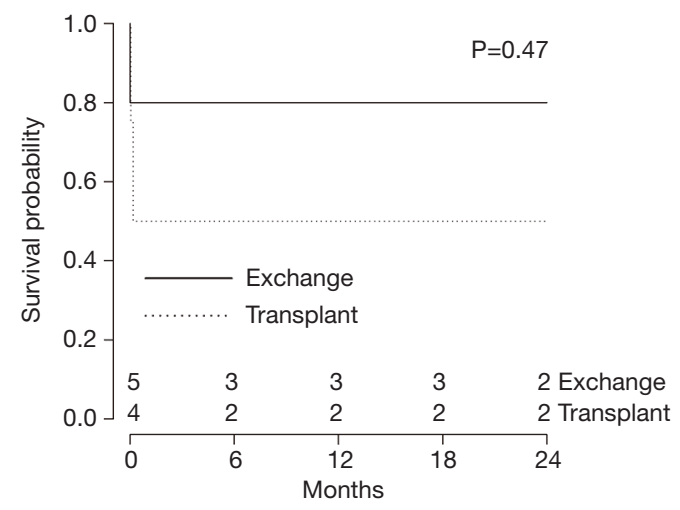

Figure 3 Kaplan-Meier survival analysis of patients with LVADassociated endocarditis who underwent LVAD exchange as compared to those who underwent heart transplantation. LVAD, left ventricular assist devices.

underwent heart transplantation following development of LVAD-associated endocarditis (LVAD exchange: $80.0 \%$ $v s$. heart transplantation: 66.7\%; $\mathrm{P}=0.23$ ) (Table 5). KaplanMeier survival analysis of patients with LVAD-associated endocarditis who underwent LVAD exchange compared to those who underwent heart transplantation is shown in Figure 3.

\section{Discussion}

LVAD-associated endocarditis can occur either due to inoculation of organisms at the time of implantation, infection of driveline site ascending into the pump-pocket, or due to bloodstream infection from other sources (16). Although rare, it is associated with significant morbidity and mortality, with studies reporting up to $50 \%$ mortality in patients with LVAD-associated endocarditis $(17,18)$. Both patient factors and the device factors play a significant role in its pathogenesis. Patient-specific factors include malnutrition, prolonged hospitalization, use of broadspectrum antibiotics, immunosuppressive medication and presence of a central venous catheter, whereas devicespecific factors include duration of the LVAD support, presence of percutaneous lead of the device, presence of turbulent blood flow inside the device, material and characteristics of the device surface in contact with the blood.

In the present systematic review, compared to patients with P-LVADs, CF-LVAD patients appeared to be resistant to early development of LVAD-associated endocarditis. There was a trend towards high survival observed amongst patients who underwent additional surgical intervention as compared to those treated with antibiotics alone, with no difference amongst surgical device exchange as compared to heart transplantation.

The median time from LVAD implantation to development of endocarditis was 91 days. The nearsignificant $(\mathrm{P}=0.05)$ trend towards longer duration to development of LVAD-associated endocarditis in patients with CF-LVAD as compared to P-LVAD may be explained by the decreased susceptibility of endocarditis in CFLVAD patients observed may be due to decreased turbulent flow, lack of valves, smaller surface area of CF-LVAD components, and advanced materials used $(19,20)$.

Hemodynamics of the LVAD device has an important role in the colonization of bacteria on the device surface in contact with the blood (21). Increased turbulence of blood flow in P-LVADs has been shown to induce shear stress associated apoptosis of neutrophils, thus preventing innate immunity to inhibit microorganism implantation and colonization of the device (22). Furthermore, patients with LVADs are more susceptible to opportunistic infections as compared to the general population due to decreased T-cell mediated immunity secondary to a defective proliferative response and activation-induced apoptosis (23). This decrease in immunity has been hypothesized to predispose patients with LVADs to bacterial and fungal biofilm formation on the LVAD surface.

LVAD-associated endocarditis can present without any clinical signs, and may only be diagnosed after an 
explanted device culture is done at the time of autopsy or device exchange (18). Clinical features and complications in LVAD-associated endocarditis are similar to those of infective endocarditis, with fever and leukocytosis being the most common presentation. Other manifestations include cerebrovascular accidents or mycotic aneurysms caused by embolization. When the two groups (CF-LVAD vs. P-LVAD) were compared, there was no significant difference in the rate of cerebrovascular accidents.

As there are no established standardized diagnostic criteria to diagnose LVAD-associated endocarditis, a combination of tests can be used to increase diagnostic yield (24). These tests include blood cultures, imaging studies and cultures from explanted LVADs (Table 1). A blood culture may be negative due to recent antibiotic use. Radiographic studies including transesophageal echocardiography has limited diagnostic value due to its difficulty in visualizing the inner surface of the LVAD (25). These factors render the diagnosis of LVAD-associated endocarditis difficult. Imaging modalities such as leukocyte scintigraphy and single-photon emission computer tomography (SPECT/CT) can be used in determining the anatomic location and extent of LVAD-associated endocarditis (26). Cultures from explanted LVADs in the presence of clinical signs were associated with the highest diagnostic yield and were seen in the majority of patients (Table 1).

LVAD-associated endocarditis is associated with high mortality and treatment should be initiated based on clinical suspicion (18). Management options for LVAD-associated endocarditis include use of antibiotics, antifungals, LVAD exchange, and heart transplantation $(27,28)$. In the present study, there was a trend towards higher survival amongst patients undergoing surgical intervention as compared to antibiotics alone, though this did not reach statistical significance. It has been previously suggested that when compared with LVAD exchange, heart transplantation is the more effective treatment option for LVAD-associated endocarditis $(27,28)$. However, in the present study, there was no demonstrable difference in long-term survival amongst patients who underwent LVAD exchange as compared to heart transplantation for LVAD-associated endocarditis (Figure 3). This may be due to our study being underpowered to detect differences as well as the morbidity associated with both procedures-whereby, patients who underwent heart transplantation have to be immunosuppressed, leading to further susceptibility to opportunistic infections. As well, LVAD exchange with replacement of an infected device with a new device is associated with a high infection relapse rate and major morbidity and mortality (29). As such, due to the shortage of donor hearts available for transplantation, LVAD exchange may be considered a viable alternative.

\section{Limitation}

This systematic review has several key limitations and must be interpreted with care. First, data was extracted mainly from case reports and case series with very few retrospective studies. Despite having applied stringent inclusion criteria, we are unable to rule out the possibility of missing some important cases aggregated in larger series, given that some individual patient data were unavailable in these series. This led to a small number of patients, as such, the study is underpowered.

There was heterogeneity in diagnostic testing and management of LVAD-associated endocarditis amongst the studies. We were unable to account for the prevalence of pathogens at a particular site within an LVAD device, as not all LVAD devices were explanted and cultured to confirm the diagnoses. Finally, when comparing the data of patients who underwent device exchange versus heart transplantation, Kaplan-Meier analysis should be interpreted with caution, especially at the endpoints since follow-up was not documented in two of the six patients who underwent heart transplantation. We acknowledge that this heterogeneity in study population is a fundamental limitation that cannot be addressed due to inability to extract sufficient detail from the pooled data. Moreover, the heterogeneity in result precludes broad generalization into prognostic terms.

\section{Conclusions}

Compared to P-LVADs, CF-LVADs appear to be resistant to early development of LVAD-associated endocarditis. There was a trend towards high survival observed amongst patients who underwent additional surgical intervention as compared to those treated with antibiotics alone. In terms of surgical intervention, there was no demonstrable difference in long-term survival amongst patients who underwent LVAD exchange as compared to heart transplantation for LVAD-associated endocarditis. Advantages of additional surgical intervention $v s$. medical therapy alone deserves further exploration to determine its applicability in CF-LVADs. 


\section{Acknowledgments}

None.

\section{Footnote}

Conflicts of Interest: The authors have no conflicts of interest to declare.

\section{References}

1. Haeck ML, Hoogslag GE, Rodrigo SF, et al. Treatment options in end-stage heart failure: where to go from here? Neth Heart J 2012;20:167-75.

2. Rose EA, Gelijns AC, Moskowitz AJ, et al. Long-Term Use of a Left Ventricular Assist Device for End-Stage Heart Failure. N Engl J Med 2001;345:1435-43.

3. Aaronson KD, Eppinger MJ, Dyke DB, et al. Left ventricular assist device therapy improves utilization of donor hearts. J Am Coll Cardiol 2002;39:1247-54.

4. Bleszynski PA, Luc JG, Schade P, et al. Current State and Future Perspectives of Energy Sources for Totally Implantable Cardiac Devices. ASAIO J 2016;62:639-45.

5. Tchantchaleishvili V, Luc JGY, Cohan CM, et al. Clinical Implications of Physiologic Flow Adjustment in Continuous-Flow Left Ventricular Assist Devices. ASAIO J 2017;63:241-50.

6. Cheng A, Williamitis CA, Slaughter MS. Comparison of continuous-flow and pulsatile-flow left ventricular assist devices: is there an advantage to pulsatility? Ann Cardiothorac Surg 2014;3:573-81.

7. Tchantchaleishvili V, Luc JGY, Haswell J, et al. Subxiphoid Exchange of HeartMate II Left Ventricular Assist Device. ASAIO J 2017;63:414-8.

8. Lee S, Fukamachi K, Golding L, et al. Left ventricular assist devices: from the bench to the clinic. Cardiology 2013;125:1-12.

9. Miller LW, Pagani FD, Russell SD, et al. Use of a Continuous-Flow Device in Patients Awaiting Heart Transplantation. N Engl J Med 2007;357:885-96.

10. Slaughter MS, Rogers JG, Milano CA, et al. Advanced Heart Failure Treated with Continuous-Flow Left Ventricular Assist Device. N Engl J Med 2009;361:2241-51.

11. Martin BJ, Luc JGY, Maruyama M, et al. Driveline Site Is Not a Predictor of Infection After Ventricular Assist Device Implantation. ASAIO J 2018;64:616-22.

12. Nienaber JJC, Kusne S, Riaz T, et al. Clinical Manifestations and Management of Left Ventricular
Assist Device-Associated Infections. Clin Infect Dis 2013;57:1438-48.

13. Kirklin JK, Naftel DC, Kormos RL, et al. Second INTERMACS annual report: more than 1,000 primary left ventricular assist device implants. J Heart Lung Transplant 2010;29:1-10.

14. Schaffer JM, Allen JG, Weiss ES, et al. Infectious complications after pulsatile-flow and continuous-flow left ventricular assist device implantation. J Heart Lung Transplant 2011;30:164-74.

15. Nienaber JJ, Kusne S, Riaz T, et al. Clinical manifestations and management of left ventricular assist device-associated infections. Clin Infect Dis 2013;57:1438-48.

16. Vilchez RA, McEllistrem MC, Harrison LH, et al. Relapsing bacteremia in patients with ventricular assist device: an emergent complication of extended circulatory support. Ann Thorac Surg 2001;72:96-101.

17. Oz MC, Argenziano M, Catanese KA, et al. Bridge experience with long-term implantable left ventricular assist devices. Are they an alternative to transplantation? Circulation 1997;95:1844-52.

18. Simon D, Fischer S, Grossman A, et al. Left ventricular assist device-related infection: treatment and outcome. Clin Infect Dis 2005;40:1108-15.

19. Goldstein DJ. Worldwide experience with the MicroMed DeBakey Ventricular Assist Device as a bridge to transplantation. Circulation 2003;108 Suppl 1:II272-7.

20. Baddour LM, Bettmann MA, Bolger AF, et al. Nonvalvular cardiovascular device-related infections. Circulation 2003;108:2015-31.

21. Arrecubieta C, Asai T, Bayern M, et al. The role of Staphylococcus aureus adhesins in the pathogenesis of ventricular assist device-related infections. J Infect Dis 2006;193:1109-19.

22. Shive MS, Salloum ML, Anderson JM. Shear stressinduced apoptosis of adherent neutrophils: a mechanism for persistence of cardiovascular device infections. Proc Natl Acad Sci U S A 2000;97:6710-5.

23. Ankersmit HJ, Tugulea S, Spanier T, et al. Activationinduced T-cell death and immune dysfunction after implantation of left-ventricular assist device. Lancet 1999;354:550-5.

24. Thyagarajan B, Kumar MP, Sikachi RR, et al. Endocarditis in left ventricular assist device. Intractable Rare Dis Res 2016;5:177-84.

25. Nurozler F, Argenziano M, Oz MC, et al. Fungal left ventricular assist device endocarditis. Ann Thorac Surg 2001;71:614-8. 
26. Litzler PY, Manrique A, Etienne M, et al. Leukocyte SPECT/CT for detecting infection of left-ventricularassist devices: preliminary results. J Nucl Med 2010;51:1044-8.

27. Prendergast TW, Todd BA, Beyer AJ 3rd, et al. Management of left ventricular assist device infection with heart transplantation. Ann Thorac Surg 1997;64:142-7.

Cite this article as: Patel S, Rizvi SSA, Choi JH, Horan DP, Weber MP, Maynes EJ, Luc JGY, Aburjania N, Entwistle JW, Morris RJ, Massey HT, Tchantchaleishvili V. Management and outcomes of left ventricular assist device-associated endocarditis: a systematic review. Ann Cardiothorac Surg 2019;8(6):600-609. doi: 10.21037/acs.2019.04.04
28. Poston RS, Husain S, Sorce D, et al. LVAD bloodstream infections: therapeutic rationale for transplantation after LVAD infection. J Heart Lung Transplant 2003;22:914-21.

29. Gordon SM, Schmitt SK, Jacobs M, et al. Nosocomial bloodstream infections in patients with implantable left ventricular assist devices. Ann Thorac Surg 2001;72:725-30. 